\title{
BIOÉTICA Y EDUCACIÓN SUPERIOR EN MÉXICO
}

\author{
Pedro César Cantú Martínez ${ }^{1}$
}

Resumen: Este artículo se refiere a la situación de la bioética en México, caracterizando las circunstancias y el contexto social en que se imparte en las instituciones de educación superior. El desarrollo de programas educativos en este nivel y el de educación continua en materia de bioética se ha convertido en una necesidad para responder a las demandas sociales mediante la formación de recursos humanos que contribuyan a mejorar la sociedad.

Palabras clave: bioética, educación superior, instituciones educativas, México

\section{Bioethics and superior education in Mexico}

Abstract: This article refers to the bioethics status in Mexico, characterizing the circumstances and the social context in which it is imparted in institutions of superior education. The development of training programs in this level and continuous education in bioethics topics have become a necessity in order to respond to social demands through training human resources which contribute to enhance society.

Key words: bioethics, superior education, education institutions, Mexico

\section{Bioética e educaçáo superior no México}

Resumo: Este artigo se refere à situação da bioética no México, caracterizando as circunstâncias e o contexto social em que é oferecido nas instituições de educação superior. O desenvolvimento de programas educativos neste nível e no de educação continuada em matéria de bioética se converteu numa necessidade para responder às demandas sociais mediante a formação de recursos humanos que contribuam para melhorar a sociedade.

Palavras-chave: bioética, educação superior, instituiçốes educativas, México

\footnotetext{
${ }^{1}$ Facultad de Salud Pública y Nutrición, Universidad Autónoma de Nuevo León, Monterrey, México Correspondencia: pedro.cantum@uanl.mx
} 


\section{Introducción}

Hacia el final del siglo XX en México, de acuerdo con Cruz López y Cruz López(1), se produjo una significativa modificación en la distribución territorial de la población en el país, como también del sector económico, en el que es notorio un preeminente desarrollo concéntrico en torno a los centros urbanos, con una alta concentración de la población urbana que hoy alcanza la cifra del $77,8 \%$ de los 112 millones de habitantes con los que cuenta México(2). Esto ha implicado un desequilibrio en el acceso a beneficios sociales para la población, ya que es en estos centros urbanos donde principalmente se lleva a cabo la actividad económica.

No obstante el progreso en México, en numerosos sectores de carácter social en los últimos años, los desafíos en el punto particular de desarrollo social aún son innegables en el país. Esto lleva a un compromiso por los tres órdenes de gobierno (federal, estatal y municipal) —conjuntamente con el Congreso de la nación- de llevar una labor permanente que permita aumentar los niveles de prosperidad y bienestar, así como lograr la legitimación de los derechos sociales de la población mexicana(3). Lo anterior ha justificado que la Comisión Económica para América Latina y el Caribe (CEPAL)(4), en 2012, ubique a México en la posición número ocho entre los países de la región con más pobreza, lo que implica que el $36,3 \%$ de la población mexicana se encuentre en esta condición, donde el país con el más alto registro de pobreza es Honduras, con un 67,4\%. Esta situación se incrementó a 37,1\% en el país, de acuerdo con el último reporte de la CEPAL en 2013(5).

Lo anterior obedeció, según mencionó en 2012 el Consejo Nacional de Evaluación de la Política de Desarrollo Social (CONEVAL)(3), al escenario acaecido durante 2009, un año con muchas dificultades para la economía del mundo y que produjo en nuestro país una pérdida de aproximada de 172 mil empleos y que llevó a la contracción del PIB en un 6\%, además de la pérdida adquisitiva del ingreso por trabajo, suscitado durante el periodo del 2008 al 2010, que se aunó al incremento en el precio de los alimentos, con cifras, de acuerdo con el CONEVAL(3), que superaron la propia inflación durante el lapso de 2007 a 2010. Se ha observado la fragilidad de México ante los eventos de orden internacional, al poner a prueba las políticas sociales, como aquellas relacionadas con el empleo y con las prestaciones sociales, que lamentablemente orillaron a muchos mexicanos a la pobreza.

En el rubro de educación, en México se consigna este derecho en el Artículo 3 de la Constitución Política de los Estados Unidos Mexicanos, de acuerdo con la última reforma publicada en el Diario Oficial de la Federación el 20 Diciembre del 2013, la cual indica: "Todo individuo tiene derecho a recibir educación. El Estado -Federación, Estados, Distrito Federal y Municipiosimpartirá educación preescolar, primaria, secundaria y media superior. La educación preescolar, primaria y secundaria conforman la educación básica; esta y la media superior serán obligatorias" (6:4). Para fortalecer esta política pública en México, que refiere a Programas y Acciones de Educación, se destinó durante 2011 la cantidad de 210.237,62 millones de pesos, lo que representó una variación del $27 \%$ de acuerdo con el presupuesto ejercido durante el año 2008 , que fue de 164.285,93 millones de pesos(3).

Lo precedente plantea desafíos a las instituciones de educación superior, propios de la dinámica ejercida por la política pública vigente, pero además, de acuerdo con Tamez Guerra y Martínez Rizo(7:157-158), se apunta que: "En las últimas décadas se han mostrado con mayor intensidad dos de los fenómenos que inciden de manera determinante sobre la naturaleza y magnitud de la demanda de servicios de educación superior: la diversificación creciente y la transformación acelerada de los distintos campos del conocimiento y la actividad humana...".

El contexto de nación descrito anteriormente determina que en México se hayan creado escenarios sociales de inequidad, de no justicia y una fisura significativa, para lograr una conveniente condición de bienestar y de salud de la población mexicana, lo que, asociado a los problemas de gobernabilidad e inestabilidad social existente, impacta negativamente en la calidad de vida de la población. Dada esta situación y conforme a los avances de carácter tecnocientífico, resulta inevi- 
table hacer esfuerzos por impulsar la bioética en las instituciones superiores en México, con el fin de crear valores sociales que incidan en nuestra sociedad y que impulsen la formación integral de los profesionistas, ya que estos se integrarán una realidad nacional plagada de asimetrías y, como señala Cantú Martínez(8), es necesario no solo formar profesionistas, sino construir seres humanos que actúen honestamente, objeten la inequidad y la falta de justicia social.

\section{El imperativo de la bioética en la educación superior}

La bioética se despliega en una nueva asignatura que sirve de reflexión profunda para el juicio de toda acción humana(8). Actualmente, la bioética "es clave para trabajar por un cambio de paradigma que transforme las bases de la cultura y la educación predominantes en el mundo global en el que vivimos"(9:1).

Este concepto es más que pertinente hoy, si observamos que en el escenario internacional provoca extrañeza y confusión, como marca De la Isla(10), cuando se observa que en el ámbito tecnocientífico la sociedad en el mundo no ha logrado resolver las eventualidades más connotadas para los seres humanos, no obstante la forma superlativa en que la ciencia y la tecnología han avanzado en el entorno universal. Se reconoce en las instituciones de educación superior (IES) una de las instancias que ha impulsado el saber tecnocientífico buscando el desarrollo de la colectividad social, por lo cual ha logrado una gran notabilidad(8), pero más es esta notabilidad por el papel que juega en la implantación de valores y los roles que juegan los profesionistas egresados de las IES, al hacer uso de estos saberes en la sociedad, tratando de alzar a un profesionista "que responda lo más virtuosamente posible a su condición humana e impulse lo más decentemente humano en las variadas circunstancias que se suscitan en la vida humana y se contribuya con valores para humanizar la sociedad"(8:29). En este marco bioético, una de las aristas sería conferir de bienes y servicios de calidad, buscando maximizar los beneficios a toda la población por parte de los profesionistas egresados.
Sin embargo, el panorama social que se yergue frente a los egresados de las IES está plagado de muestras de dominación por unos pocos y de violencia social, en el que se ha excluido a millones de personas de estos beneficios y, como señala Bermúdez de Caicedo(11:37), "el capitalismo puede sobrevivir con que el $20 \%$ de la población posea el $80 \%$ de los recursos y el $80 \%$ de la población, los más pobres, sólo tengan el 20\% de estos. ¿Es esto compatible con la bioética?”. Como señala De la Isla(10): “¿Qué decir de la educación pensada como fórmula de éxito económico para la competencia globalizada, para obtener más y mejores conocimientos vendibles? ¿№ es ésta la forma dominante, la gran mentira de la educación?"

Aunque en el plano de los sectores educativos, especialmente en el superior, estos cuestionamientos son una realidad que predomina, las discusiones sobre las problemáticas socioambientales con un contenido bioético se han introducido recientemente(12), y hoy es inexcusable no realizarlas. La inserción de la bioética en el plano de la educación superior examina primeramente las formas para abandonar la formación profesional sustentada solo en el utilitarismo, como razón instrumental del quehacer profesional, y en segundo término favorece los aspectos de carácter humanístico de la preparación profesional en asistencia a la población(13), porque tiene que ver con el beneficio común.

Lo anterior fortalece la tarea obligatoria y fundamental de las IES, que es proveer un entorno situacional interno que ayude al progreso y potenciación de todas las capacidades humanísticas, intelectuales y técnicas que en ellas se construyen por los académicos y estudiantes; pero, particularmente, es a través de estos últimos, los estudiantes, que se constituirán en los futuros profesionistas y emisarios para llevar este beneficio a la colectividad social. Así, la inserción de la bioética como asignatura en la educación superior permitiría a los egresados de las IES "tomar decisiones libres y responsables ante situaciones o dilemas, acordes al contexto y respetando las diferencias de un mundo pluralizado"(11:36), donde se privilegie la equidad y justicia social para todo ser humano. 


\section{Contexto de la educación superior en México}

Comprender y detallar los cambios que se sus suscitan en materia de educación superior a través de las IES en México se constituye en un desafío considerable, ya que estos cambios son el resultado de la interacción de elementos que obran y circulan, en una correspondencia estrecha, entre el ámbito interno de las IES y el escenario socioambiental en el cual prevalecen. Entre estos factores externos hallamos las circunstancias socioeconómicas nacionales existentes, las políticas educativas gubernamentales de turno y aquellas de resolución internacional que marcan pautas para reorientar objetivos y actividades, con el fin de responder a determinados momentos o situaciones históricas. Es así que destacaremos solo algunos momentos que nos parecen relevantes de esta trayectoria.

México cuenta con una dilatada historia sobre el surgimiento de las IES, que se remonta a 1551, cuando, bajo el impulso de Fray Juan de Zumárraga, se instaura la Real y Pontificia Universidad de México, que constituye lo que ahora conocemos como la Universidad Nacional Autónoma de México(14). Esta fue erigida en el mismo período que la Universidad Nacional Mayor de San Marcos en Perú, tras el surgimiento inicial de la Universidad de Santo Tomás de Aquino en Republica Dominicana, en 1538(15).

México, al igual que un gran número de países de Latinoamérica y el Caribe, ha experimentado un aumento en la oferta de educación superior, reflejándose también en la diversidad del tipo de instituciones que esta educación superior contempla. La Ley para la Coordinación de la Educación Superior en México, que data de 1978, tipifica en su artículo 3 a la educación superior como aquella "que se imparte después del bachillerato o de su equivalente. Comprende la educación normal, la tecnológica y la universitaria e incluye carreras profesionales cortas y estudios encaminados a obtener los grados de licenciatura, maestría y doctorado, así como cursos de actualización y especialización" (16:1). La educación superior debe atender las prioridades sociales de carácter nacional, regional y estatal, por tanto, hoy en día, la educación superior en México se erige como uno de los dispositivos ineludibles para lograr el progreso del país.
Lo anteriormente mencionado es apuntado por del Val(17), al exteriorizar que la educación superior se establece como uno de los artífices para el crecimiento económico, así como construir una sociedad más incluyente y mejorar los niveles de bienestar de la población mexicana. En este mismo marco legal antes mencionado, particularmente en el artículo 4, se citan las funciones que las IES en nuestro país deben impulsar, como son la docencia, investigación y difusión de la cultura, señalando que estas actividades deben conservar entre sí, una consonancia y complementariedad.

Otro de los sucesos más relevantes fue la constitución, el 25 de marzo de 1950, de la Asociación Nacional de Universidades e Instituciones de Educación Superior en México (ANUIES(18). En ese momento, como advierte Pallan Figueroa(18:14), la "población del país era de 25 millones, la matrícula de educación superior ascendía a $32 \mathrm{mil}$ estudiantes ( 3 mil 800 de ellos mujeres, 12\%), las 26 instituciones fundadoras representaban $75 \%$ de la matrícula nacional en educación superior y, de los jóvenes en edad de concurrir las aulas universitarias, sólo $1,3 \%$ tenía acceso efectivo a las mismas". Esto contrasta con el escenario actual, en el que México cuenta ahora con una población total de 112.336.538 de habitantes, de acuerdo al INEGI(2); donde, para el ciclo escolar 20112012, la Secretaría de Educación Pública en México(19) reporta una matrícula escolarizada para la educación superior de 3.161 .195 alumnos, de los cuales el 3,85\% corresponde al nivel técnico superior o profesional asociado, 4,23\% a normallicenciatura, $84,68 \%$ a licenciatura universitaria y tecnológica, y 7,24\% a posgrado. No considerando el posgrado, la matrícula en educación superior atañe al 29,4\% de la población de 19 a 23 años de edad en el país. Por otra parte, esta cifra se eleva a $32,8 \%$ cuando contempla la matrícula de alumnos en modalidad mixta y no escolarizada. ANUIES está ahora constituida por 175 IES, tanto públicas como particulares, de todo el país, y su principal función es fortalecer los ámbitos de la docencia, la investigación, la extensión de la cultura y los servicios(20).

Por otra parte, en materia de educación superior en México, durante las décadas de los ochenta y noventa del siglo pasado, prevaleció un escenario de múltiples restricciones de carácter presupues- 
tario que, aunado a una falta de cobertura en el ámbito de educación superior, impidió satisfacer la demanda de la población joven entre las edades de 19 y 23 ańos, por lo tanto, el gobierno de México condujo una estrategia de diversificación de la educación superior, para impulsar el crecimiento de la oferta a través de la IES privadas, pero también promovió otra alternativa de educación superior de orden público, como fue el perfil tecnológico(17). Según la Secretaría de Educación Pública en México(19), la matrícula de educación superior se distribuye mayormente en IES con un sustento autónomo, alcanzando una cifra de $37,1 \%$, siguiendo las IES con sostenimiento particular $(31,7 \%)$. El resto corresponde a soporte económico de carácter estatal y federal, con un 17,8 y un $13,4 \%$ respectivamente.

México, miembro de la Organización para la Cooperación y el Desarrollo Económico (OCDE), que agrupa a 34 países, menciona que el "porcentaje de egresados universitarios sigue siendo muy inferior a la media de la OCDE, de 39\%, los niveles de educación superior entre los jóvenes han aumentado 6 puntos porcentuales entre 2000 (17\%) y $2011(23 \%)$, y ya superan a los de Austria (21\%), Brasil (13\%), Italia (21\%) y Turquía (19\%)"(21:1).

\section{Antecedentes y situación actual de la bioética en México}

Durante siglos la educación superior ha dado muestra fehaciente de su alta capacidad para transformarse y favorecer el progreso de toda sociedad. Particularmente porque, en la situación actual, se reconoce que la sociedad mexicana, reflejo del contexto internacional, vive una profunda crisis de valores, que no solo ha trascendido a los aspectos socioeconómicos, sino también a espacios de moralidad, concibiendo el orden moral como Latapí Sarre(22:61): "el orden moral, que tiene su propia especificidad y un lugar central en el desarrollo humano, es el orden del uso responsable de la libertad, pues no otra cosa es la moral".

En consecuencia, la formación en valores de los educandos de educación superior se erige como la adquisición de un conjunto de cualidades deseables en diferentes esferas del desarrollo humano, esencialmente aquellas que recurren al empleo responsable y consciente de su libertad como individuos. Con esto, la formulación de proyectos y reformas académicas para renovar y actualizar la dinámica institucional de las IES en la formación de recursos humanos es imprescindible en México, dado el diverso y cambiante escenario social en el que estarán insertos, en el que, simultáneamente, ha acaecido una creciente polarización y pauperización socioeconómica en distintas regiones del país, que se yergue en un sinnúmero notable de desigualdades, exclusiones y luchas sociales.

Por esta razón, es importante acoger la enseñanza de la bioética que, de acuerdo con la Declaración Universal sobre la Bioética y los Derechos Humanos(23), exhorta en su artículo 23 a los países miembros a impulsar la instrucción a todos los niveles educativos y la formación académica en bioética. $\mathrm{Al}$ proseguir en este transitorio análisis nacional, es pertinente determinar el contexto en el que se encuentra México respecto del contexto regional en materia de bioética, ya que nuestro país no se exime de este marco referencial cultural e ideológico. De acuerdo con Mainetii, existen tres consideraciones iniciales que posicionan la bioética en Latinoamérica, esto corresponde a los ámbitos biomédico, biojurídico y biopolítico, que dan pie a los principales debates que subsisten en la región; indicando además que entre estas posturas coexiste un punto de encuentro de carácter filosófico común, y señala "sus actuales proyecciones es el término bios, entendido como $z o e ́$ (vida biológica) y biotós (calidad de vida) para la medicina, bios prosopón (vida personal) para el Derecho, bios politikós (vida pública) para la política" (24:116).

Lo anterior es de suma importancia porque marca el derrotero que ha seguido la bioética tanto en su posicionamiento como en su desarrollo en México, el cual obedece a estas tres vertientes discursivas mencionadas, donde la biomédica cuenta con una dilatada representación en las facultades de ciencias de la salud en las IES, pero también en otros espacios, como los medico-hospitalarios, colegios, academias, asociaciones y sociedades científicas, por citar algunas(25). En este derrotero de la vertiente biomédica en México, durante 1980 el país fue sede de la Conferencia Internacional del Consejo Internacional de Organizacio- 
nes Internacionales de Ciencias Médicas(26), que atendió la propuesta de creación de normas internacionales para la investigación biomédica en sujetos humanos; aunque aún en el discurso de las ciencias de la salud en México no prevalecía la bioética en ese momento, como señalan Cano Valle y Neri Vela(25:264). "Esto contrastaba con la difusión mundial creciente de la Bioética a partir de la octava década del siglo pasado".

Lo anteriormente citado llevó a que en 1989 se fundará el Grupo de Estudios en Bioética, cuyo objetivo central giraría alrededor del estudio, investigación y difusión de la bioética en el país, en el marco de las atribuciones otorgadas por el Consejo de Salubridad Nacional(25). Este Grupo dio paso a la instalación más adelante, en 1992, de la Comisión Nacional de Bioética, la cual es permanente desde 2000(27) y hoy actúa como órgano descentralizado de la Secretaría de Salud, con capacidad operativa y técnica de forma independiente. Hoy estas acciones se ven ratificadas y fortalecidas mediante el Programa Sectorial de Salud (2013-2018), publicado en el Diario Oficial de la Federación el 12 de diciembre del 2013, que indica en la estrategia 6.5: situar a la bioética como política de gestión y de desarrollo del Sistema Nacional de Salud Universal(28). Sin embargo, estos esfuerzos de carácter nacional, dejan entrever una ausencia de la educación en bioética en otros espacios disciplinares de la educación superior, como si esto fuera solo exclusivo de los profesionales de la salud, aseverando Téllez-López(29:107): "No se han desarrollado programas de bioética que integren a todas las ciencias, conocimientos y actividades de investigación, orientados hacia la formación de hombres y mujeres íntegros, con valores sólidos, de respeto a la dignidad humana, a la calidad de vida y a los derechos humanos".

No obstante que las IES en México ofrecen una gama de cursos de bioética en facultades, principalmente de la salud y otras como derecho, filosofía, por citar algunas, en nuestro país existe una oferta educativa, aunque no dilatada, de programas de posgrado y educación continua en educación superior que, indistintamente de sus enfoques, abordan esta temática en el país. Después de una búsqueda en internet, observamos que, en el escenario nacional, esta formación superior ofrece programas de grados académicos, como doctorado, maestría, especialidad y diplomaturas. Existen, al menos tras nuestra indagación, tres programas que ofrecen el grado de Doctorado en Bioética, diez de Maestría en Bioética, cuatro de Especialidad en Bioética y siete de Diplomaturas en Bioética, oferta concentrada en siete ciudades, como son Ciudad de México, Monterrey, Guadalajara, Querétaro, Mérida y León (Cuadro 1).

Cuadro 1. Programas de educación superior y de educación continua en bioética que se imparten en México

\begin{tabular}{|c|c|c|}
\hline Institución & Programa(s) & Ciudad \\
\hline $\begin{array}{l}\text { Instituto de In- } \\
\text { vestigaciones en } \\
\text { Bioética }\end{array}$ & $\begin{array}{l}\text {-Doctorado en } \\
\text { Bioética } \\
\text {-Maestría en Bioé- } \\
\text { tica } \\
\text {-Especialidad en } \\
\text { Bioética } \\
\text { - Diplomado en } \\
\text { Bioética }\end{array}$ & Monterrey \\
\hline $\begin{array}{l}\text { Universidad } \mathrm{Na}- \\
\text { cional Autónoma } \\
\text { de México }\end{array}$ & $\begin{array}{l}\text {-Doctorado en } \\
\text { Bioética } \\
\text {-Maestría en Bioé- } \\
\text { tica } \\
\text {-Diplomado en } \\
\text { Bioética }\end{array}$ & $\begin{array}{l}\text { Ciudad de } \\
\text { México }\end{array}$ \\
\hline $\begin{array}{l}\text { Universidad } \\
\text { Anáhuac-México } \\
\text { Norte }\end{array}$ & $\begin{array}{l}\text {-Doctorado en } \\
\text { Bioética } \\
\text {-Maestría en Bioé- } \\
\text { tica }\end{array}$ & $\begin{array}{l}\text { Ciudad de } \\
\text { México }\end{array}$ \\
\hline $\begin{array}{l}\text { Centro de Estudios } \\
\text { E Investigaciones } \\
\text { en Bioética }\end{array}$ & $\begin{array}{l}\text {-Maestría en Bioé- } \\
\text { tica } \\
\text {-Especialidad en } \\
\text { Bioética } \\
\text {-Diplomado en } \\
\text { Bioética }\end{array}$ & Guadalajara \\
\hline $\begin{array}{l}\text { Colegio de Bioética } \\
\text { de Nuevo León }\end{array}$ & $\begin{array}{l}\text {-Maestría en Bioé- } \\
\text { tica } \\
\text {-Especialidad en } \\
\text { Bioética } \\
\text {-Diplomado en } \\
\text { Bioética }\end{array}$ & Monterrey \\
\hline
\end{tabular}




\begin{tabular}{|l|l|l|}
\hline $\begin{array}{l}\text { Universidad Pana- } \\
\text { mericana-Campus } \\
\text { México }\end{array}$ & $\begin{array}{l}\text {-Maestría en Bioé- } \\
\text { tica } \\
\text {-Especialidad en } \\
\text { Bioética }\end{array}$ & $\begin{array}{l}\text { Ciudad de } \\
\text { México }\end{array}$ \\
\hline $\begin{array}{l}\text { Instituto Politéc- } \\
\text { nico Nacional }\end{array}$ & $\begin{array}{l}\text {-Maestría en Bioé- } \\
\text { tica }\end{array}$ & $\begin{array}{l}\text { Ciudad de } \\
\text { México }\end{array}$ \\
\hline $\begin{array}{l}\text { Centro de Inves- } \\
\text { tigación Social } \\
\text { Avanzada }\end{array}$ & $\begin{array}{l}\text {-Maestría en Bioé- } \\
\text { tica }\end{array}$ & Querétaro \\
\hline $\begin{array}{l}\text { Universidad Ana- } \\
\text { huac-Mayab }\end{array}$ & $\begin{array}{l}\text {-Maestría en Bioé- } \\
\text { tica }\end{array}$ & Mérida \\
\hline $\begin{array}{l}\text { Colegio de Bioé- } \\
\text { tica y Terapia de } \\
\text { Jalisco }\end{array}$ & $\begin{array}{l}\text {-Maestría en Bioé- } \\
\text { tica }\end{array}$ & Guadalajara \\
\hline $\begin{array}{l}\text { Universidad } \\
\text { Autónoma Metro- } \\
\text { politana. Unidad } \\
\text { Xochimilco }\end{array}$ & $\begin{array}{l}\text {-Diplomado en } \\
\text { Bioética }\end{array}$ & $\begin{array}{l}\text { Ciudad de } \\
\text { México }\end{array}$ \\
\hline $\begin{array}{l}\text { Universidad de } \\
\text { Guanajuato }\end{array}$ & $\begin{array}{l}\text {-Diplomado en } \\
\text { Bioética }\end{array}$ & León \\
\hline $\begin{array}{l}\text { Colegio de Bioética } \\
\text {-Diplomado en } \\
\text { Bioética }\end{array}$ & $\begin{array}{l}\text { Ciudad de } \\
\text { México }\end{array}$ \\
\hline
\end{tabular}

\section{Conclusiones}

Se reconoce a las IES como las instancias que en México trabajan ceñidamente con el conocimiento, tanto para generarlo como aplicarlo, así como en la formación de recursos humanos de nivel superior. Los educandos se constituyen en su materia prima, lo que da pertinencia a su actividad sustantiva. Pero también las IES se encuentran fuertemente influidas por el entorno socioambiental en que subsisten, aunado también el marco internacional, que es consubstancial y del cual no pueden substraerse.

Ante esto, la bioética se erige como espacio en el que encontrar un sustrato fundamental para la transformación del orden social. Al mismo tiempo, la bioética podría coadyuvar al fortalecimiento de las identidades culturales locales, como también al de los valores humanos, aquellos conceptos de carácter universal, comunes a toda sociedad.

Por otra parte, el esfuerzo para seguir impulsando y para ampliar la oferta de enseñanza de la bioética en las IES en México debe buscar una mayor lucidez y responsabilidad en estas instituciones, porque la coherencia del discurso y la acción, trasmitida a través de la formación disciplinar de los educandos, debe ser la respuesta a los problemas que sobrevienen del mal desarrollo. Los cambios de orden social se asientan en el círculo virtuoso hermenéutico, que sucede de manera espontánea entre las IES que posibilitan la reflexión y el debate respecto de la realidad que, en términos sociales, subsiste en el entorno que les rodea.

\section{Referencias}

1. Cruz López Y, Cruz López, AC. La educación superior en México. Tendencias y desafíos. Avaliaçâo: Revista da Avaliação da Educação Superior 2008; 13(2): 293-311.

2. INEGI. Cuadro resumen. Indicadores de demografía y población, 2010. Consulta 21 de enero de 2104. Consultado en: http://www3.inegi.org. $\mathrm{mx} /$ sistemas/temas/default.aspx?s=est\&c=17484

3. Consejo Nacional de Evaluación de la Política de Desarrollo Social (CONEVAL). Avances y Retos de la Politica de Desarrollo Social en México 2012. México: CONEVAL; 2012.

4. Comisión Económica para América Latina y el Caribe (CEPAL). Panorama Social de América Latina 2012. Santiago de Chile: ONU-CEPAL; 2012.

5. Comisión Económica para América Latina y el Caribe (CEPAL). Panorama Social de América Latina 2013. Santiago de Chile: ONU-CEPAL; 2013.

6. Gobierno de México. Constitución Política de los Estados Unidos Mexicanos. Diario Oficial de la Federación, 20 de diciembre de 2013.

7. Tamez Guerra RS, Martínez Rizo F. Las reformas que necesita la educación mexicana. México: No Venal; 2012.

8. Cantú-Martínez PC. La bioética en las Instituciones de Educación Superior en el contexto de la sustentabilidad. Ciencia UANL 2014; 17(65): 24-30.

9. Quintanas A. V. R. Potter: una ética para la vida en la sociedad tecnocientífica. Revista Electrónica Sinéctica 2009; 32 : 1-5.

10. De la Isla C. Ética y Universidad. Estudios 2004, 2(69): 7-18. 
11. Bermúdez de Caicedo C. Necesidad de la bioética en la educación superior. Acta Bioethica 2006; 12(1): 35-40.

12. Rama C. Ética y educación superior en el contexto de la mercantilización. Universidades 2004; 28:3-12.

13. Linares Márquez P, Fraile Laiz B. Bioética y Universidad en América Latina. Propuesta de una estructura para la formación integral profesional, Revista Colombiana de Bioética 2012; 7(2): 120-133.

14. Maya A. La educación superior en México. Una mirada a su historia. Revista AAPAUNAM Academia, Ciencia y Cultura 2012; 4(2): 104-107.

15. UDUAL. Historia de las universidades de América Latina. México: Unión de Universidades de América Latina; 1999.

16. Gobierno de México. Ley para la Coordinación de la Educación Superior en México. Diario Oficial de la Federación, 29 de diciembre de 1978.

17. Del Val E. Educación superior, ciencia y tecnología en México. Tendencias, retos, prospectiva. Revista de la Universidad de México 2011; 87 (mayo): 11-23.

18. Pallán Figueroa C. La ANUIES y su presencia en la educación superior nacional. Boletín Confluencia 2010; 8(171): 13-19.

19. Secretaría de Educación Pública Sistema educativo de los Estados Unidos Mexicanos. Principales cifras ciclo escolar 2011 2012. México: DGPP/SEP; 2012.

20. Asociación Nacional de Universidades e Instituciones de Educación Superior (ANUIES). Acerca de ANUIES 2013, Fecha de Consulta 10 Diciembre del 2013. Consultado en: http://www.anuies.mx/content.php?varSectionID=2

21. OCDE. Panorama de la Educación 2013, México 2013, Fecha de Consulta: 15 de Diciembre 2103. Consultado en: http://www.oecd.org/edu/Mexico_EAG2013\%20Country\%20note\%20(ESP).pdf

22. Latapí Sarre P. Valores y educación. Ingenierías 2001; 4(11): 59-69.

23. UNESCO. Declaración universal sobre bioética y derechos humanos. París: UNESCO; 2005.

24. Mainetti JA. Educación en Bioética: Tres discursos de la Bioética en América Latina: Bio-médico, Bio-jurídico y Biopolítico. En: Vidal SM, (ed.) La Educación en Bioética en América Latina y el Caribe: Experiencias realizadas y desafios futuros. Montevideo: Oficina Regional de Ciencia de la UNESCO para América Latina y el Caribe; 2012: 111-118.

25. Cano Valle F, Neri Vela RA. Educación en bioética en México. En: Vidal SM, (ed.) La Educación en Bioética en América Latina y el Caribe: Experiencias realizadas y desafios futuros. Montevideo: Oficina Regional de Ciencia de la UNESCO para América Latina y el Caribe; 2012: 261-278.

26. Porter JK, De la Escosura G. Panorama bioético en México. En Organización Panamericana de la Salud (ed.) Bioética: Temas y perspectivas. Washington: OPS-Publicación Científica No 527; 1999: 171-178.

27. Comisión Nacional de Bioética. Antecedentes de la Comisión Nacional de Bioética 2013. Fecha de Consulta: 30 de enero 2014. Consultado en: http://www.conbioetica-mexico.salud.gob.mx/interior/antecedentes.html

28. Gobierno de México. Programa Sectorial de Salud 2013-2018. Diario Oficial de la Federación, 12 de diciembre de 2013.

29. Téllez López AM. La enseñanza de la ética en las escuelas de educación superior. En: Morales González, JA, Nava Chapoy G, Esquivel Soto J, Díaz Pérez LE. Principios de ética, bioética y conocimientos del hombre. Pachuca: Universidad Autónoma del Estado de Hidalgo; 2011: 107-109.

Recibido: 13 de agosto de 2014

Aceptado: 25 de septiembre de 2014 